BNL-113448-2017-CP

\title{
A New Prototype X-ray Fluorescence Detector System with Silicon Drift Detector Array
}

\author{
W. Chen, G. DeGeronimo, D. Elliott, G. Giacomini, A. J. Kuczewski, \\ J. Mead, D. Pinelli, A. K. Rumaiz, D. P. Siddons, G. Smith and E. O. Vernon \\ Presented at the 2016 IEEE Nuclear Science Symposium (NSS)/Medical Imaging \\ Conference \\ IEEE Nuclear Sciences \& Society \\ October $29^{\text {th }}$ through November $5^{\text {th }}$
}

November 2016

Physics Department

Brookhaven National Laboratory

\author{
U.S. Department of Energy \\ USDOE Office of Science (SC), \\ High Energy Physics (HEP) (SC-25)
}

Notice: This manuscript has been authored by employees of Brookhaven Science Associates, LLC under Contract No. DE- SC0012704 with the U.S. Department of Energy. The publisher by accepting the manuscript for publication acknowledges that the United States Government retains a non-exclusive, paid-up, irrevocable, world-wide license to publish or reproduce the published form of this manuscript, or allow others to do so, for United States Government purposes. 


\section{DISCLAIMER}

This report was prepared as an account of work sponsored by an agency of the United States Government. Neither the United States Government nor any agency thereof, nor any of their employees, nor any of their contractors, subcontractors, or their employees, makes any warranty, express or implied, or assumes any legal liability or responsibility for the accuracy, completeness, or any third party's use or the results of such use of any information, apparatus, product, or process disclosed, or represents that its use would not infringe privately owned rights. Reference herein to any specific commercial product, process, or service by trade name, trademark, manufacturer, or otherwise, does not necessarily constitute or imply its endorsement, recommendation, or favoring by the United States Government or any agency thereof or its contractors or subcontractors. The views and opinions of authors expressed herein do not necessarily state or reflect those of the United States Government or any agency thereof. 


\title{
A New Prototype X-ray Fluorescence Detector System with Silicon Drift Detector Array
}

\author{
W. Chen, G. DeGeronimo, D. Elliott, G. Giacomini, A. J. Kuczewski, J. Mead, D. Pinelli, A. K. Rumaiz, D. P. \\ Siddons, G. Smith and E. O. Vernon
}

\begin{abstract}
A new Maia detector is being currently developed, consisting of an array of 384 silicon drift detectors (SDDs), each having an area of $1 \mathrm{~mm}^{2}$. The detector features a new double metal scheme that supplies biases to the pixel SDD rings. All 384 anodes of SDDs are meant to be connected to individual electronic channels. This effort was motivated by the need of improving the energy resolution and the throughput of the existing Maia detectors whose pixels are based on p-in-n diodes. In order to use the existing circuit boards and back-end electronics that have already developed for the original Maia, the SDD-Maia detector was designed with an identical footprint, after replacing the p-in-n diodes with SDDs. The design, fabrication and performance of a first prototype detector will be presented.
\end{abstract}

\section{INTRODUCTION}

$\mathrm{T}$ HE Maia detector, which has been developed over the last five years, is used for fluorescence spectroscopy at synchrotrons. The original Maia detector consists of an array of 384 silicon p-in-n diodes ( $20 \times 20$ pixel array minus $4 \times 4$ pixels been removed in the central hole), each pixel diode having an area of $1 \mathrm{~mm}^{2}$, and connected to an individual electronic channel. Several of these diode-based Maia systems are in operation at synchrotrons around the world. The synchrotron X-rays are usually directed onto the sample under test and a detector with spectroscopic capability is used to identify the fluorescence X-rays emitted from the sample, which are naturally lower in energy than that of the incoming $\mathrm{X}$-rays. Determination of the fluorescence energy can lead to identification of elemental composition of the sample. A mechanical drawing of the experimental set-up is shown in Figure 1. One of the goals of the diode-based Maia development has been to achieve high count-rates by maximizing the coverage of the detector array solid-angle, instead of increasing the flux. In this way the sample damage can be minimized. The diode-based Maia detector array uses an annular, "back-scattering" geometry, where the beam passes through the detector located upstream of the sample. The annular configuration enables a large solid-angle without imposing severe restrictions on the sample size or the scale of scanning. At its optimum, the diode based Maia detector has a full width half maximum (FWHM) noise in each channel of about $250 \mathrm{eV}$, at a shaping time of $4 \mu \mathrm{s}$. These two critical

Manuscript received Dec. 6, 2016.

W. Chen*, G. DeGeronimo, D. Elliott, G. Giacomini, A. J. Kuczewski, J. Mead, D. Pinelli, A. K. Rumaiz, D. P. Siddons, G. Smith and E. O. Vernon are employed by the Brookhaven National Laboratory (BNL), Upton, NY 11973 USA (*631-344-4213, weichen@bnl.gov). parameters determine the detectors' ability to identify two fluorescence lines close together in energy, and the acquisition rate $[1,2]$.

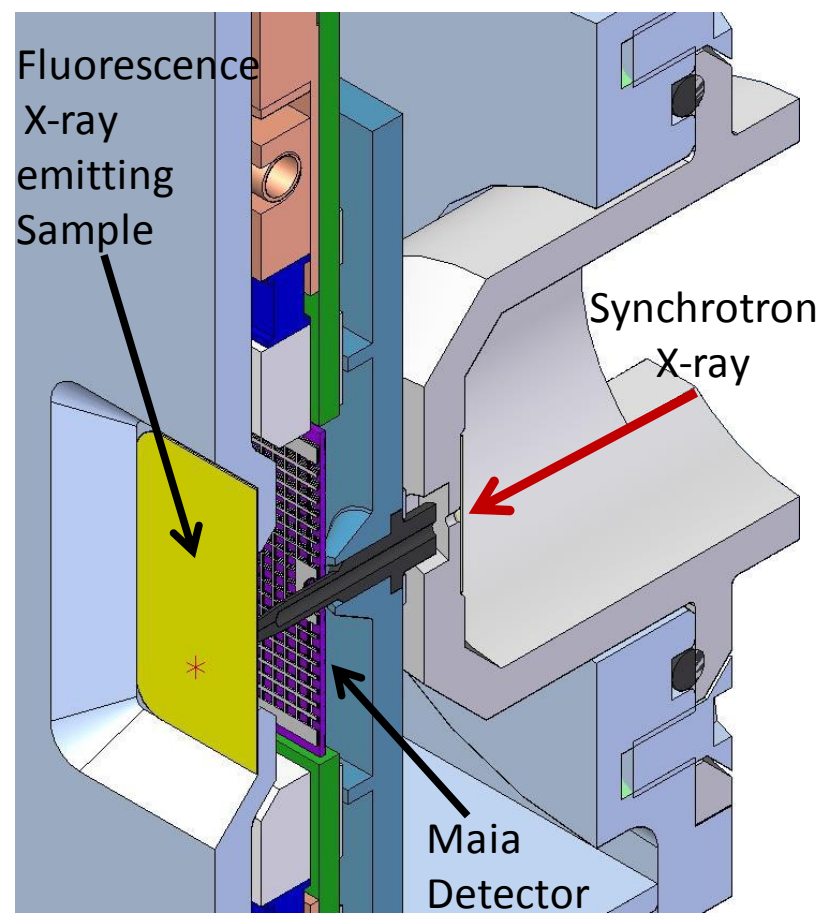

Fig. 1. Incident synchrotron X-rays pass through the sensor's central hole and hit the sample. The back scattered fluorescence is detected by the sensor.

In order to improve the energy resolution and the throughput, and to continue the use of the same circuit boards and back-end electronics that have already been developed, we designed a new Maia detector with a footprint identical to the original Maia, after replacing the diode sensors with silicon drift detectors (SDDs) [3]. The SDD features a small capacitance due to its small area anode compared to a diode of the same sensitive area, and hence yields a lower electronic noise when connected to a charge preamplifier. This will thus permit shorter shaping times and an improved energy resolution, and thus increase the throughput per unit time. Based on our previous experience with SDD arrays [4-7], where we achieved an electronic noise of about $162 \mathrm{eV}$ at an energy of $6 \mathrm{keV}$ at a shaping time of $1 \mu \mathrm{s}$, we expect that the new Maia will reduce the experimental measurement time by four times, while lowering the noise. 


\section{Design Features}

The design of the Drift Maia detector array consists of a 20 $\times 20$ array of $1 \mathrm{~mm}^{2}$ SDD sensors, minus a central group of 4 $\mathrm{x} 4$ SDDs removed to accommodate the passage of the X-ray beam through the detector and to the sample. A detail of the design is shown in Figure 2. For the design convenience, the 384 array is formed by four identical arrays of 96 SDDs, each being rotated 90 degree from each other. In the wafer, there are also 32, 8, 4 pixel arrays as test structure; they are the smaller version of the main 384 arrays. The shape of each individual pixel is square in order to coincide with the footprint of the original Maia detector. Each SDD pixel is made of 8 concentric "rings" that supply a voltage gradient to form the drift field within the silicon. In the wafer layout, there are two of such large arrays, differing from the way the rings are biased: 1) by punch-through or 2) directly from a voltage source, the single ring voltages distributed by means of a second metal layer. The metal lines of the second metal layer connect the SDD rings to common bias lines located outside the active area, thus reducing the total number of bonds.

Each common bias line is bonded to an external voltage divider to get the bias from it. In order to achieve bond ability, each anode is connected to a separate staggered bonding pad on the second metal layer. Each anode is meant to be wire bonded to a designated ASIC channel. The total number of electronic channels is 384 , identical to the one of the original Maia detector.

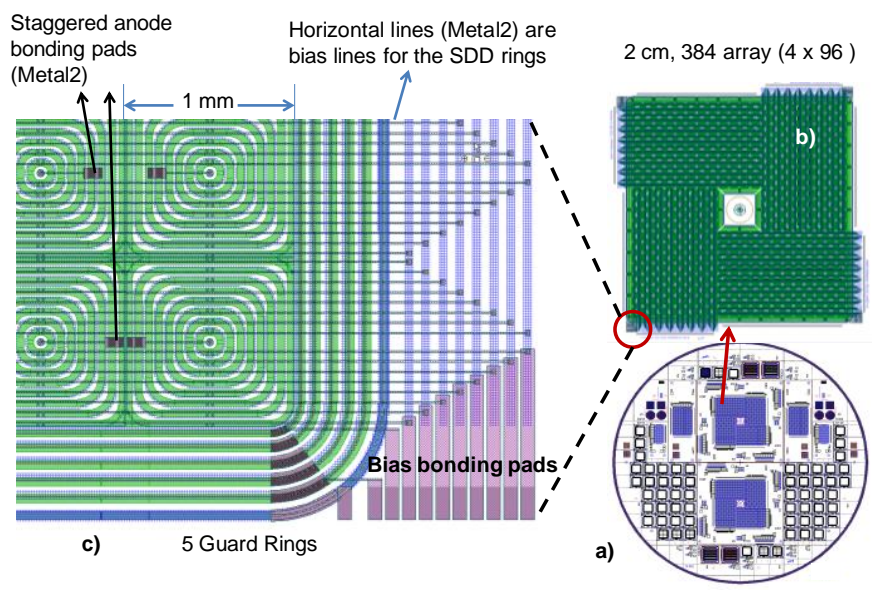

Fig. 2. a) Wafer layout. b) Design of a 384 detector array. c) A zoomed corner of the SDD Maia array with detailed design of the individual SDD detectors $\left(1 \mathrm{~mm}^{2}\right)$ which has double metal bias scheme (horizontal lines run across the SDD rings), anode bonding pads and guard rings.

\section{FABRICATION AND ASSEMBLY}

The fabrication has been done on n-type $\langle 100\rangle$, high resistivity (HR), $500 \mu \mathrm{m}$ thick wafers in the semiconductor fabrication facility of Instrumentation Division at BNL. The process consists of 11 masks. Our standard recipe to achieve a thin entrance window $[8,9]$ was applied on the backside of the detector. The double metal process for the SDD-Maia is instead new. The polyimide was used as insulation layer in between the two metal layers, which give a large thickness to keep the capacitance between the two layers to a minimum.

After completion of the fabrication the wafers have been bench tested and then laser diced into individual detectors. A 96 array was mounted on to a PC board and wire bonded to the ASICs. The Figure 3 shows the finished wafer and a diced detector with its assembly.

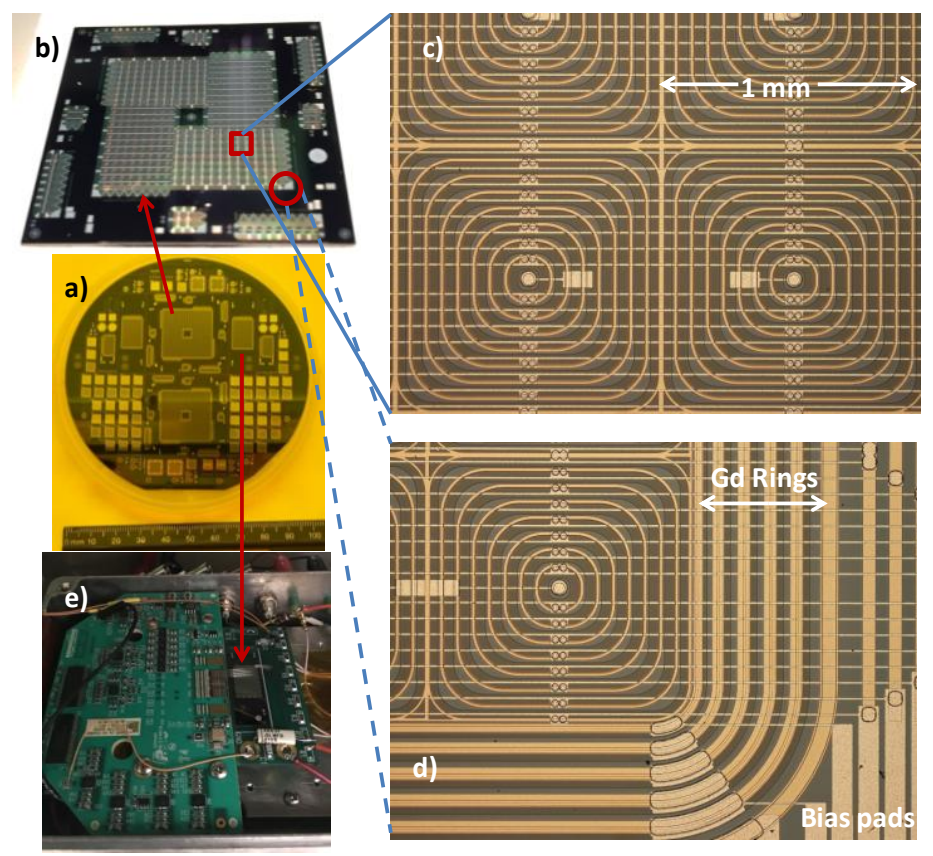

Fig. 3. Shows the finished wafer in a) with $384,96,32,8,4$ arrays; the laser diced 384 array is showing in b); the enlarged SDD pixels from random part of the diced detector is shown in c) and d); the assembled 96 array wirebonded to the ASICs on the PC board in e).

\section{RESULT AND DISCUSSION}

Measurements of the anode capacitance are shown in Figure 4 , for the two bias schemes. It can be seen that the capacitance is much higher for the SDDs biased by means of the second metal lines than for the case of punch-through biasing. In the former case, important parasitic capacitances between the two metal lines dominate the total anode capacitance. Thus, the double metal design for biasing the SDD rings is not the best choice in terms of a low noise device.

Two assembled systems, one with double-metal bias scheme and the other with the punch through bias scheme, were exposed to an uncollimated $55 \mathrm{Fe}$ source in an environment chamber. The test results are preliminary and showed on Figure 5. They show reasonable sensitivities for detecting ${ }^{55} \mathrm{Fe} \mathrm{X}$-rays for both bias schemes; all the pixels in the array are quite uniform. The FWHM of ${ }^{55} \mathrm{Fe}$ measurement at $-27^{\circ} \mathrm{C}$ for the double metal bias scheme was $250 \mathrm{eV}$ (Figure 5 a). The shaping time was $0.5 \mu \mathrm{s}$, a lower value than the original diode-based MAIA. The major noise might come from the high leakage current of the detectors, due to the malfunctioning of one of the equipment used during the fabrication, which was later understood. 


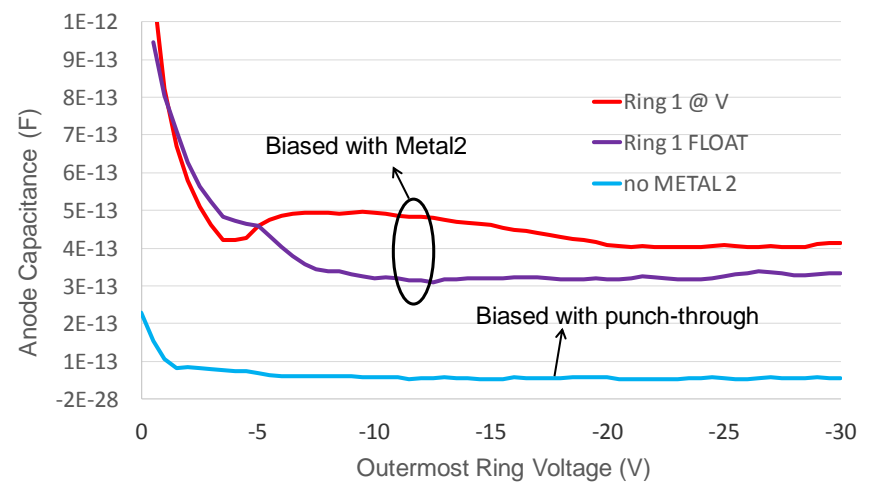

Fig. 4. Anode capacitance measurement is shown. The top two graphs are capacitances of the SDDs with double metal lines across the bias rings. One curve is the one with the first ring (surrounding anode) biased at a certain voltage, the other curve is the one with the first ring floating. The bottom graph is measured capacitance for the detector with punch through bias scheme.
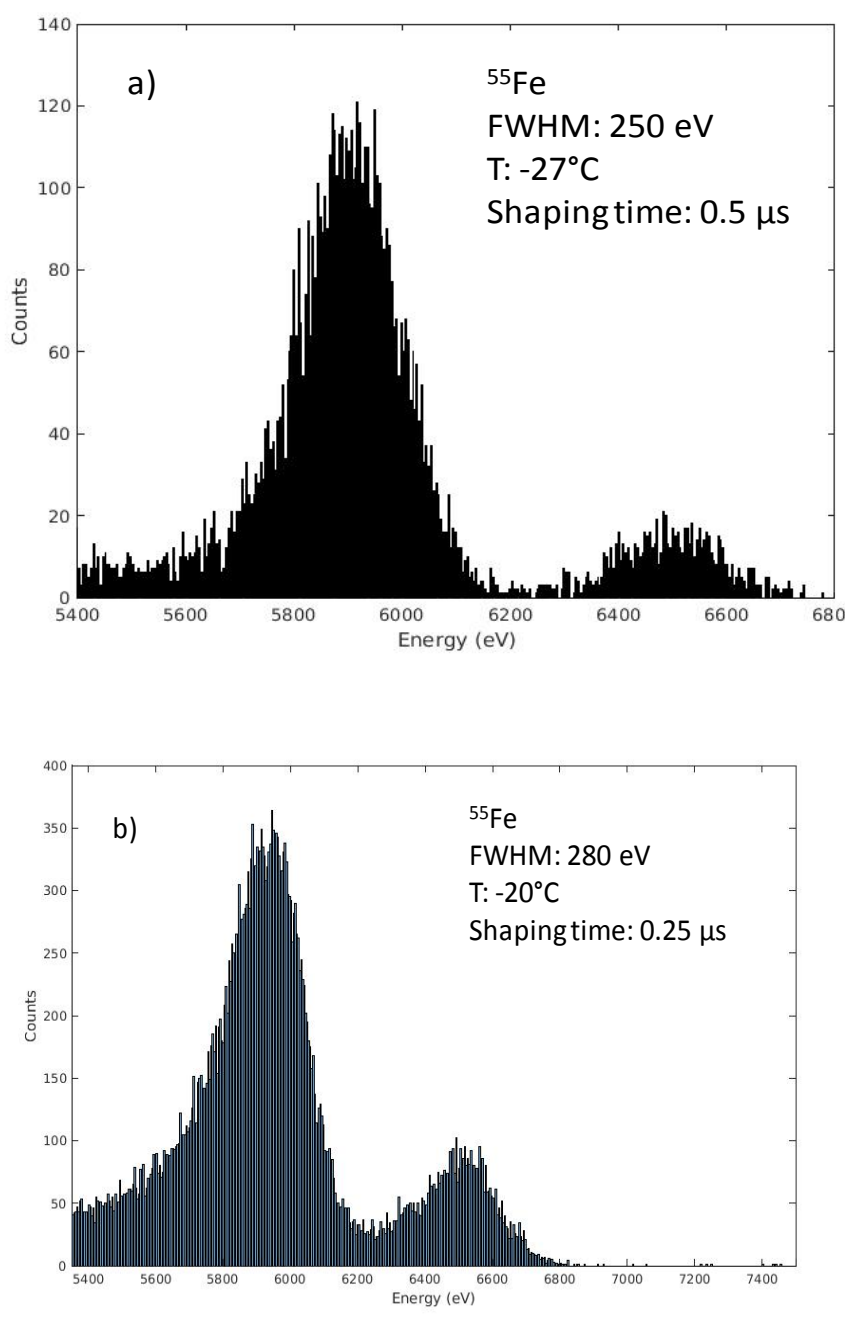

Fig. 5. ${ }^{55} \mathrm{Fe}$ spectrum measurements are shown. a) Spectrum for the detector designed with double metal bias scheme. b) Spectrum for the one with punch through bias scheme.

For the punch-through bias scheme the FWHM of ${ }^{55} \mathrm{Fe}$ measurement at $-20^{\circ} \mathrm{C}$ was $280 \mathrm{eV}$ at a shaping time was 0.25 $\mu$ s (Figure $5 \mathrm{~b}$ ). All these measurement are preliminary, and further tests are still going on.

\section{CONCLUSIONS}

A new prototype of an SDD-Maia detector array has been designed and produced. Preliminary tests have shown reasonable sensitivities for detecting ${ }^{55} \mathrm{Fe} \mathrm{X}$-rays. We do achieve the same noise performance as the diode-based MAIA detector, but at the lower shaping times, due to the lower anode capacitances. From the inputs of the results of the first fabrication batch, a new fabrication batch of detectors has been started. A modified design is needed to further improve the performances.

\section{ACKNOWLEDGEMENT}

This work was supported in part by the U.S. Department of Energy under Contract No. DE-AC02-98CH10886 and also was funded in part by the Laboratory Directed Research and Development (LDRD). The publisher by accepting the manuscript for publication acknowledges that the United States Government retains a non-exclusive, paid-up, irrevocable, world-wide license to publish or reproduce the published form of this manuscript, or allow others to do so, for United States Government purposes.

\section{REFERENCES}

[1] Siddons D P, Kirkham R, Ryan C G, De Geronimo G, Dragone A, Kuczewski A J, Li Z Y, Carini G, Beuttenmuller R, Elliott D et al. “Maia X-ray Microprobe Detector Array System”, Journal of Physics: Conference Series, Volume 499, conference 1 (http://iopscience.iop.org/1742-6596/499/1/012001)

[2] C G Ryan, D P Siddons, R Kirkham, Z Y Li, M D de Jonge4, D J Paterson, A Kuczewski et al. "Maia X-ray fluorescence imaging: Capturing detail in complex natural samples", Journal of Physics: Conference Proceeding, 2013.

[3] C Ryan, R Kirkham, D Siddons, P Dunn, J Laird, A Kuczewski, G Moorhead, G De Geronimo, P Davey, et al., "The Maia 384 Detector Array in a Nuclear Microprobe: A Platform for High Definition PIXE Elemental Imaging”, Nucl. Instrum. \& Meth. B, 268, 1899-1902 (2010) [91330]

[4] Gatti and P. Rehak, "Semiconductor Drift Chamber - an application of a novel Charge Transport Scheme”. Nucl. Instrum. \& Meth. A225 (1984) 608-614.

[5] G. De Geronimo et al., "Front-End ASIC for High Resolution X-ray Spectrometers", IEEE Nuclear Science Symposium conference Record (2007) N3-5

[6] P. Rehak et al. "Arrays of Silicon drift Detectors for an extraterrestrial X-ray spectrometer, Nucl. Instrum. \& Meth. A624 (2010) 260-264.

[7] W. Chen, G. A. Carini, G. De Geronimo, J. A. Gaskin, J. W. Keister, S. Li, Z. Li, B. D. Ramsey, D. P. Siddons, G. C. Smith and E. Verbitskaya, "Radiation effects of n-type, low resistivity, spiral Silicon Drift Detector hybrid systems"; IEEE NSS-MIC Conference Record, 1697-1701 (2011).

[8] Chen, W., Carini, G., Keister, J., Li, Z. and Rehak, P., "Development of Thin-junction Detector", IEEE Trans. Nucl. Sci. Vol. 54, No. 5, Part 2, Oct. (2007) 1842-1848.[43914]

[9] G. A. Carini, W. Chen, G. De Geronimo, J. A. Gaskin, J. W. Keister, Z. Li, B. D. Ramsey, P. Rehak and D. P. Siddon, "Performance of a ThinWindow Silicon Drift Detector X-Ray Fluorescence Spectrometer"; IEEE Trans. Nul. Sci., Vol. 56(5), p. 2843-2849 (2009) 